\title{
Platforms for Engineering Experimental Biomedical Systems
}

\author{
Matthew Mosteller \\ Institute for Systems Research \\ University of Maryland, \\ College Park, MD 20742, USA. \\ Reza Ghodssi \\ Institute for Systems Research \\ Dept. of Electrical and Computer Eng. \\ University of Maryland, \\ College Park, MD 20742, USA. \\ Mark Austin \\ Institute for Systems Research \\ University of Maryland, \\ College Park, MD 20742, USA. \\ Shah-An Yang \\ Institute for Systems Research \\ University of Maryland, \\ College Park, MD 20742, USA. \\ Copyright (C) 2012 by the Institute for Systems Research. Published and used by INCOSE with permission.
}

\begin{abstract}
The systematic design, validation, and verification of systems for biomedical experiments in laboratory and clinical applications are complex due to the highly stochastic nature of biological systems. This paper presents a platform framework for the modeling of these biological components in the context of system-level analysis that enables formal validation and verification of biomedical devices. Looking forward, the capabilities of this platform enable the development of more efficient and effective experimental biomedical systems.
\end{abstract}

\section{Introduction}

Problem Statement. Biomedical systems designed for experimental purposes are a vital aspect of today's medical field, from bench-top systems driving advances in biological science to bedside point-of-care devices in the clinical realm. Devices aiding medical researchers in advancing the science and knowledge of physiological processes allow for the continued development of new medicines and treatment methods. Similarly, devices that are capable of providing accurate diagnoses and prognoses of patients are necessary if this developing knowledge is to help clinicians improve the health and safety of future generations.

The difficulty in developing systems for the purpose of biomedical assays is complicated immensely by the variant nature of biological systems (Endy 2005). The development of biological organisms over time is dependent on a large number of factors unique to each system, including physiological processes, genetics, and environmental conditions. Thus, the same inputs to such a system do not always result in the same set of outputs, making the design, validation, and verification of biomedical devices exceedingly difficult. Furthermore, systems designed for experimental purposes in the biomedical field are becoming progressively more complicated (Csete 2002). Researchers are becoming more interested in biological processes at the molecular level in an effort to treat ailments at their source, while clinicians are desiring tools capable of faster, more accurate, and less invasive patient analysis. Due to these increases in complexity, the efficient design of biomedical systems is becoming more difficult and more costly, since current methods for system-level design are not capable of treating the highly 
stochastic properties of biological components (Endy 2005). Looking forward, it is clear that new methods for designing experimental biomedical devices at the system level will be needed if advances in medical science and treatment are to maintain or accelerate their current pace.

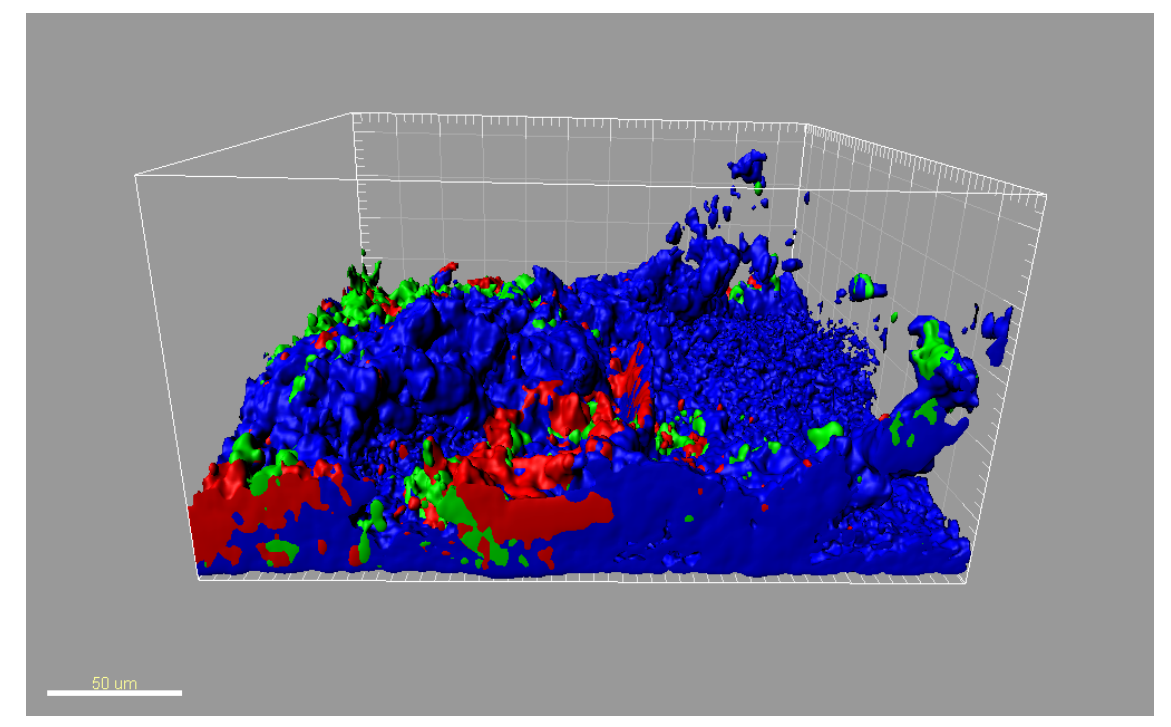

Figure 1. Surface reconstruction of a bacterial biofilm showing the highly variant nature of commonly studied biological systems

Compounding the complexity of this problem is the knowledge disconnect that must be bridged between biological and engineering domains (Endy 2005). Because of the complex nature of biological systems, understanding of this component of the larger biomedical system is typically limited to biologists and clinicians who are well versed in their fields (Oltvai 2002). For example, the growth of bacterial biofilms such as that shown in Figure 1 has been linked to as many as 65 percent of all microbial infections in the human body (Potera 1999). Such communities of microbes are extremely complex, making the prediction of their growth a limiting factor to design engineers attempting to develop new ways of treating or investigating these biological systems. Thus, while the biologist or clinician may understand the biological component of a system but not the technologies needed to address their application, the design engineer may understand the state-of-the-art but lack the background needed to properly apply these technologies.

To address this, design and development techniques must implement a method that enables one to validate and verify the system performance in the context of highly stochastic and complex biological components, thereby assimilating the two domains (Endy 2005). Drawing upon the capabilities of systems engineering tools to model systems in the design phase, the development of platforms for engineering these experimental systems is a large step towards enabling the accurate development of biomedical devices. Figure 2 expresses the methods in which these platforms would allow for the integration of the two system domains. The application space defined by the clinician or systems biologist provides the necessary knowledge to model the operation of stochastic biological components. The developed platforms then allow for the integration of such concepts with potential system architectures to create an overall design space that can effectively address the requirements of the experimental system. In order to capitalize 
upon the added capabilities of such a technique, two key tenets of this work are that (1) methods to succinctly model a breadth of biological systems must be developed and (2) these models must be able to integrate with system-level models capable of describing the performance of the entire engineering system. Current methods and techniques for experimental biomedical device development simply are not capable of such full-system modeling.

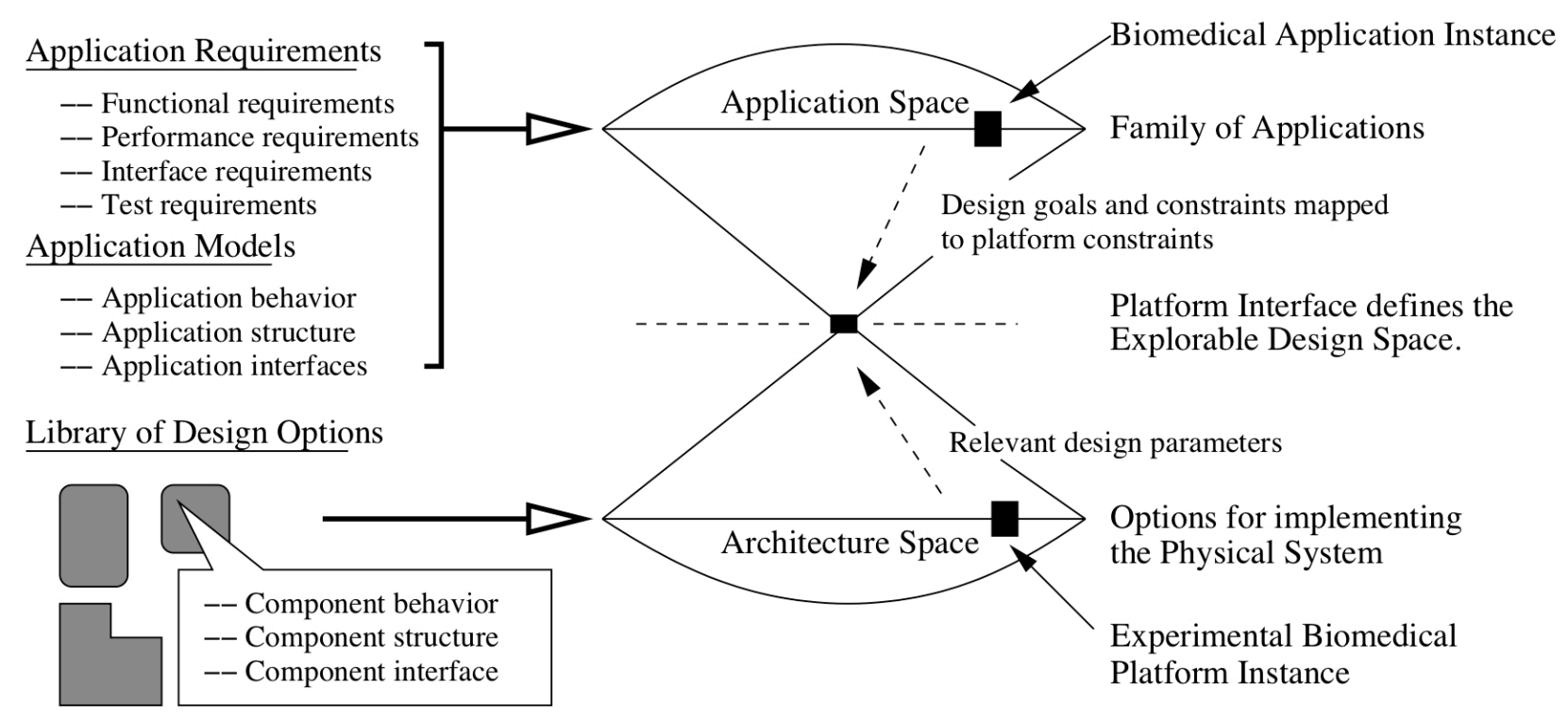

Figure 2. Application spaces motivated by biology and architecture design spaces motivated by engineering define an explorable design space

Scope and Objectives. In order to address the limitations of current design methods for experimental biomedical systems, the work presented here aims to develop platforms for the modeling, validation, and verification of device systems that contain highly stochastic biological components. By integrating models of the biological system with models of the physical engineering system, system architectures can be achieved that will perform to match the requirement specifications of the particular application. Models of biological systems can be used to analyze experimental devices that are used for applications dealing with both the advancement of medical knowledge, in which the stochastic elements are the biological systems themselves, and those pertaining to patient diagnosis and prognosis, in which the stochastic elements are the physiological responses of patients to a particular assay.

By successfully implementing such platforms, developers of these devices can ensure that results obtained from experimental tests are trustworthy representations of what the biological system state is. In another vein, these same techniques can ensure that the system will not enter an unstable state that would be otherwise unforeseeable if designing the system using traditional methods. Figure 3 reveals the ways in which these concepts can be implemented at various levels of abstraction in order to aid the development of effective, reliable, and accurate biomedical devices. 


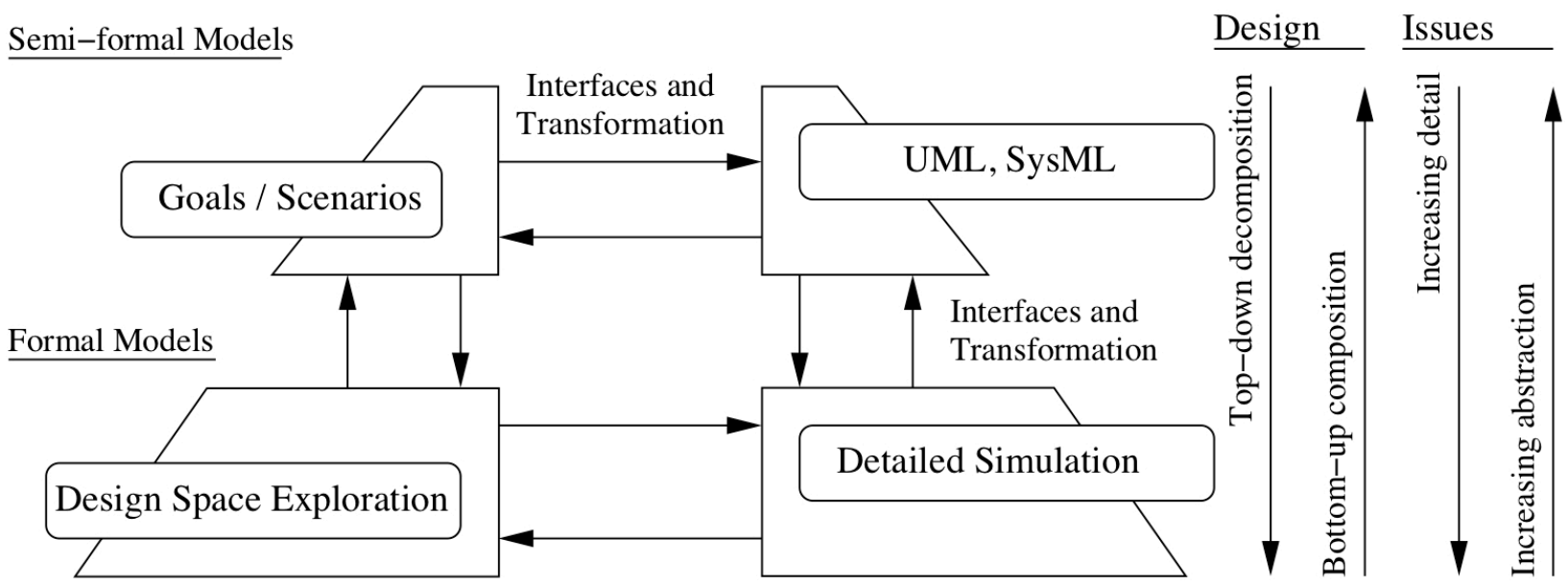

Figure 3. Abstraction as a tool for the design of biomedical Ssystems with Integrated models of stochastic biological components

Semi-formal models of the system architecture, including the requirements of the system and its proposed implementation via such modeling languages as UML or SysML can provide engineers with a high-level understanding of the system performance, thus aiding in more efficient and cost-effective redesign, validation, and verification. These semi-formal models are supported at lower levels through detailed simulations of the system, including components that embody the stochastic biological elements. By concentrating upon the integration of these stochastic components with well-defined physical systems, this work enables progress towards a level of confidence in experimental tests that was not previously obtainable for biomedical devices. The benefits of these capabilities are far-reaching across the biomedical field by enabling the development of devices that are best suited for their applications, and thus most beneficial to clinical patients, and medical researchers in search of new drugs and techniques.

\section{Experimental Biomedical Systems}

Experimental Processes. A typical experimental process in the biomedical field utilizing a device architecture is shown in figure 4. Typically, the researcher or clinician begins with a hypothesis about their subject that is developed from prior data or patient symptoms (Tomlin 2007). For a medical researcher or systems biologist, this hypothesis may involve a parameter or process that the experiment is intended to verify. Examples commonly include verification of a metabolic process, the effects of a compound on a biological system (i.e. candidate drug), or verification of unique characteristics of a certain organism. For the clinician, a hypothesis may involve a patient diagnosis or prognosis, or may be geared towards determining an effective treatment for a patient's verified medical condition. With this hypothesis in place, an experiment is begun under ideally controlled conditions. At the conclusion of the established assay, the researcher or clinician typically inspects the outcome to determine if the test was sufficiently successful or if alterations or repetition of the experiment is required. Due to the highly stochastic nature of biological systems, such a feedback process is common in order to verify experimental results. The goal of the design engineer is to develop device systems that can aid in reducing the number of iterations needed to achieve a required level of confidence in the result. 
This is especially important in clinical applications, due to the patient discomfort often associated with invasive testing (i.e. prick tests to determine skin allergies). Similarly, current medical research often utilizes high cost, low throughput methods of testing, giving strong motivation for the development of methods to limit the number of iterations needed to verify an experiment.

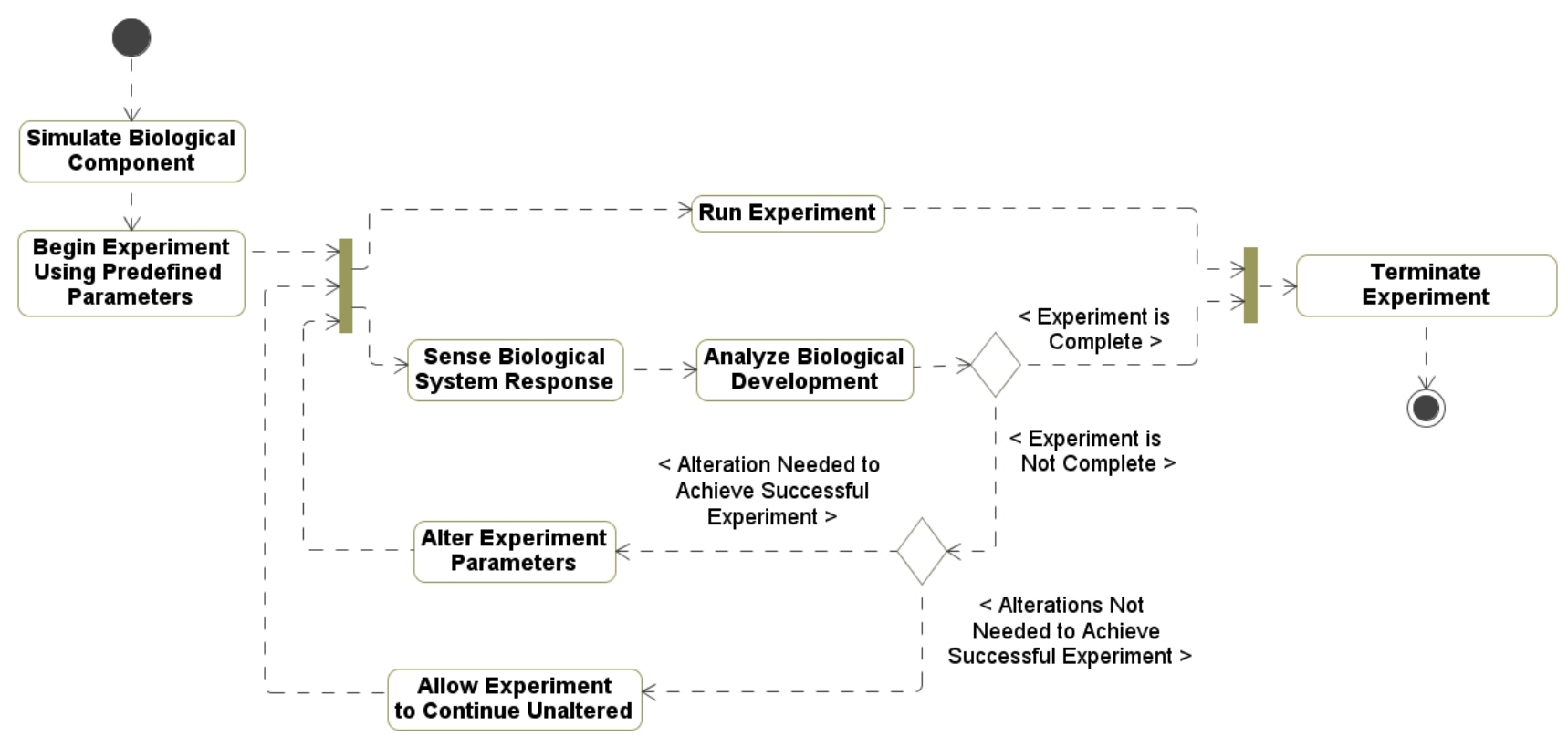

Figure 4. SysML activity diagram for process flow of typical biomedical experiments

The high cost, low throughput, and low repeatability of current biomedical testing techniques using established technologies and methods provides strong motivation for their continued development in the future. However, in order to break from the current limiting approach to biomedical device development, new techniques are needed to aid in the maturation of new device systems. The novel platforms presented here are a first step towards such methods, which can increase the overall efficiency of both device development and the operation of the devices themselves by optimizing the interactions of the biomedical elements with the physical system.

Architectures for Experimental Biomedical Systems. While the physical architecture of biomedical devices is diverse and typically suited to the needs of the particular application, most systems can be abstracted to the system architecture shown in figure 5. System inputs are typically comprised of a number of different domains, including environmental conditions and actuation or application conditions (i.e. what is done to the biological system during the experiment). Depending upon the requirements of the test, the physical system of the experimental device can take any number of forms but will typically have three distinct characteristics including: (1) a way to contain or integrate with the biological system or sample, (2) a way to control experimental conditions, and (3) a way to integrate with a sensor network for detection. The sensing mechanisms utilized for experimental devices also vary depending upon the application, though they typically aim to strike a balance between minimizing invasiveness and achieving the required detection limits and sensitivity of the application. The cumulative effect of the physical system and sensor networks' interactions with the biological element 
results in a set of potential outcomes from experiments, each of which having their own probability of occurrence. These probabilities are dependent upon the stochastic biological system, providing at the simulation level a range of statistically relevant outcomes that can be used to confirm experimental results.

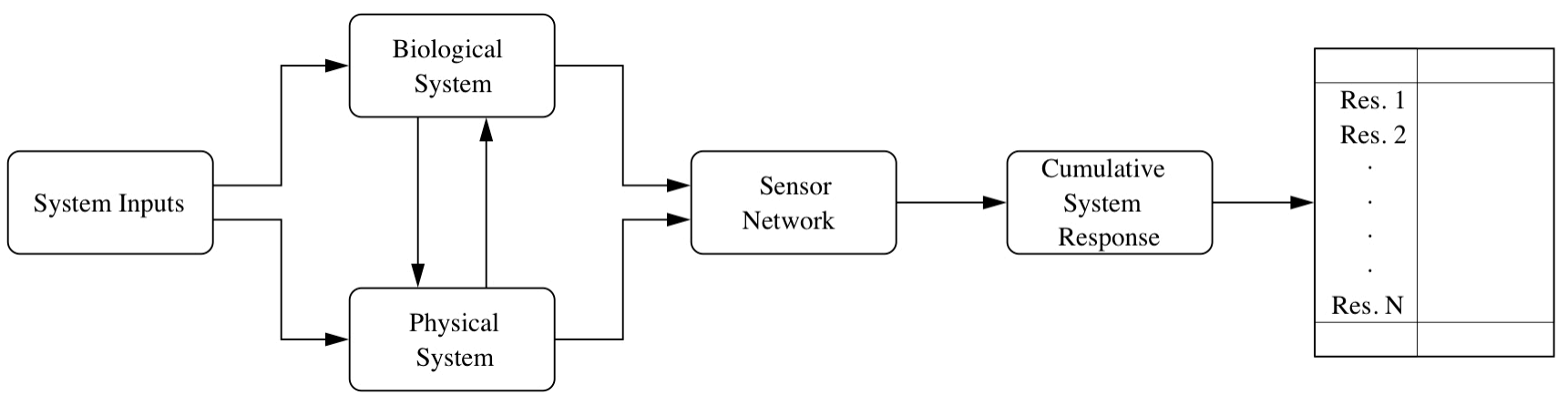

Figure 5. High-level system architecture of biomedical device performance

Most biomedical devices are constructed through a similar architecture, providing strong support for the development of generalized platforms for experimental device engineering. Figure 6 provides a high-level implementation of the system elements and their interactions at the component and subcomponent levels.

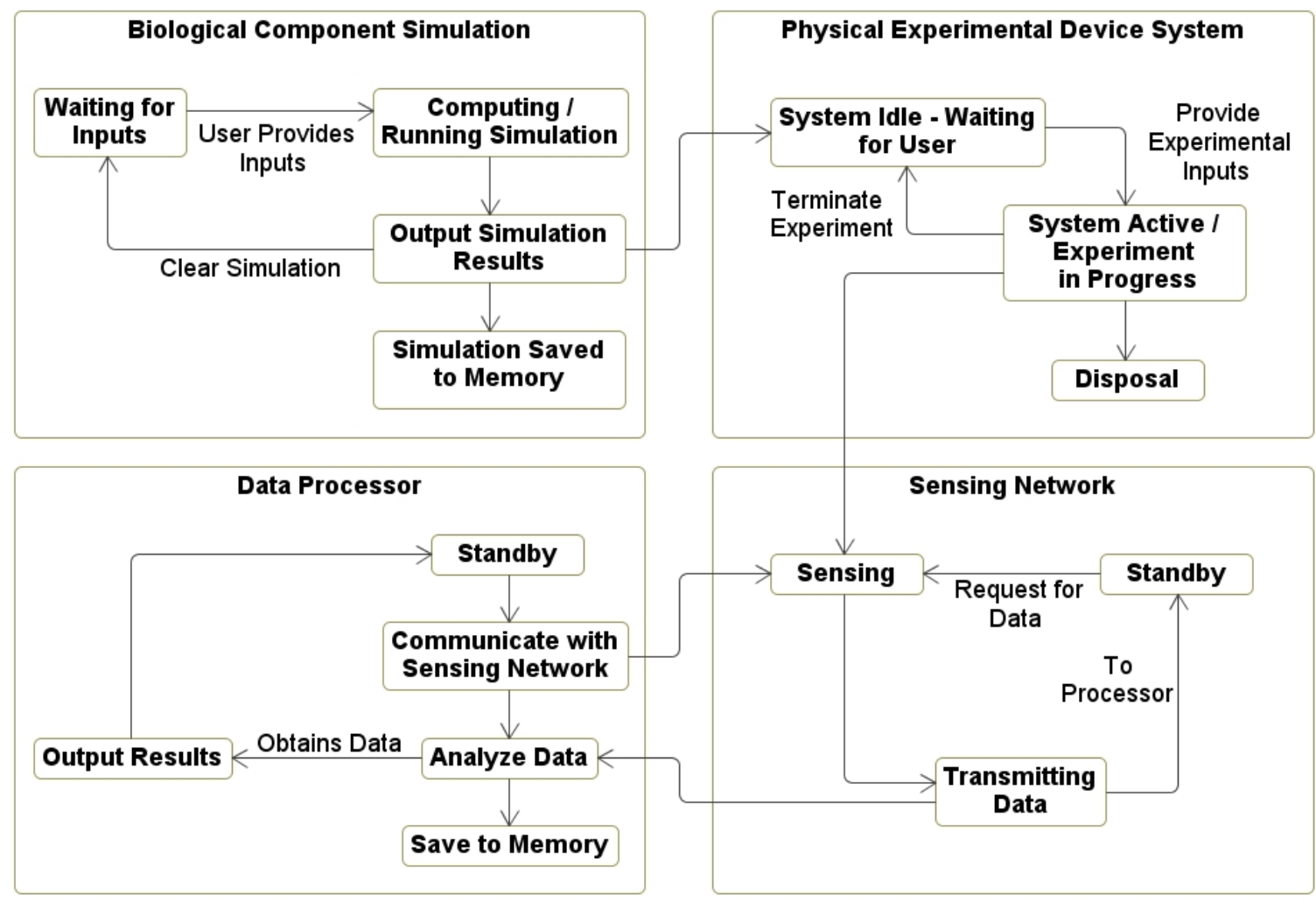

Figure 6. SysML state machines of biomedical device components 
The platforms discussed subsequently exhibit a flexible structure that can be adapted to numerous applications in the biomedical field, thus expanding the scope and influence of this work. The development of libraries of components to represent physical system and sensory network elements would aid in the efficient development of new devices and the adaptation of existing devices to new application areas. Additionally, formal platforms capable of integrating such libraries with models of the stochastic biological components enable full-system modeling that can effectively aid efficient and proper design, validation, and verification of biomedical systems. Implementation of such a platform using existing systems languages such as UML and SysML takes advantage of the mature properties of these tools, where implementing extensions to other modeling domains is a well-established practice. A tool for the succinct mathematical modeling of stochastic biomedical components would be such an extension of this platform.

\section{Mathematical Modeling of Biological Systems}

To effectively predict the operation of biomedical devices, models that efficiently and accurately predict the operation of the biological component of the system are vital. These models must be able to simulate the development of the biological system with time, as well as predict changes of the biological system due to experimental conditions. In doing so, these models can then be integrated with higher-level models of the overall experimental biomedical system to complete the platform architecture. While a number of modeling methods exist for biomedical systems that differ in their modularity, method of implementation, and overall accuracy, we focus on one particular modeling method, Markov Chains or Hidden Markov Models, as a particularly suitable method for biomedical device applications (Kim 2002, Rabiner 1986, Tomlin 2007).

Markov Chains and Hidden Markov Models. Markov Chains and Hidden Markov Models provide a method of modeling probabilistic systems with finite states (Rabiner 1986). While this method has existed for over a century, only recently have they begun to see significant use in engineering applications to understand the development of systems over time. Additionally, they have seen use in a number of other fields to model and predict the development of highly stochastic biological and population schema in order to emulate and predict their function (Baldi 1994, Durbin 2002, Kim 2002, Van Hulst 1978). A Markov Chain model can be easily visualized as a set of states, each with a probability of propagation to a future state. Figure 7 shows how a simple Markov Chain may be easily visualized.

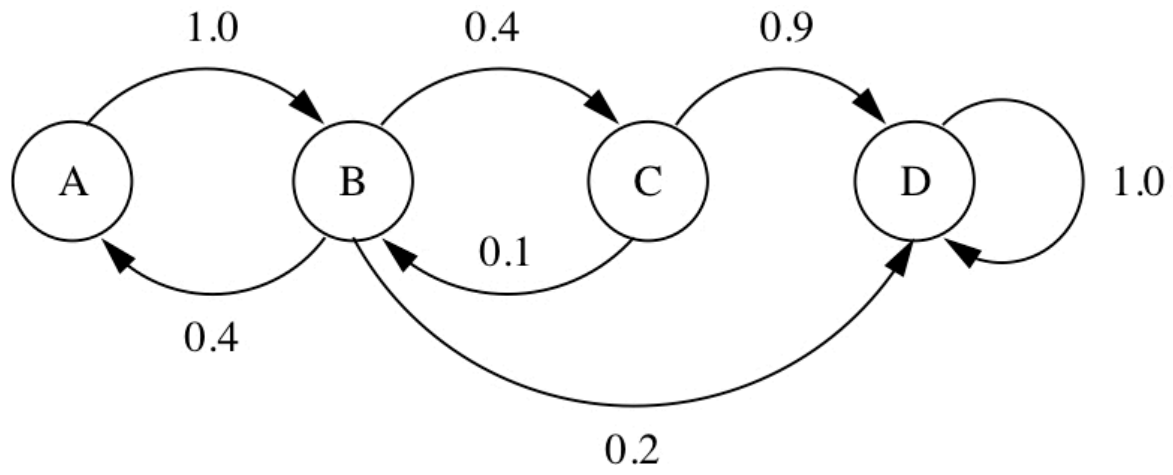

Figure 7. Markov Chain showing propagation between system states 
Each state of the Markov Chain model represents a physical system state, with the arrows from each state showing the probability of propagation to another physical system state. The sum of all propagation probabilities of each state must sum to 1.0, with feedback or steady-state operation between states also being possible. Additionally, segmentation of Markov Chain models is permitted, where the probabilities of a state's propagation may be dependent upon the current state of another related Markov Chain. Such a technique is easily scalable and enables the effective modeling of highly complex systems in a manner that is intuitive, adaptable, and quick to implement or alter (Rabiner 1986). Hidden Markov Models are an extension of the Markov Chain concept, where the Markov Chain or network of interacting Markov Chains are developed based on observed real-world performance. Behavior of a system, be it discrete in nature or a continuous spectrum, is tracked and documented, and then a Markov Model is developed to fit this system performance. This model then enables further analysis or prediction of future system functions (Rabiner 1986). The fact that these models are emergent and based upon observed system performance makes them "hidden" to the model developer, since it is not initially clear what the system states may be or how these states are related.

These characteristics make the use of Markov Chain models and Hidden Markov Models a preferred method for the representation of biological systems. Highly complex biological phenomena have already been modeled with considerable success through the use of Markov Chains. Kim, et. al. successfully developed a Markov model for the development of melanoma in patients where data was based upon the predictive relationships between 587 independent genes (Kim 2002). By determining the factors of greatest importance to the development of the biological melanoma system, a Markov model describing ten interacting genes was produced that very nearly matched the real-world development of the system (steady state convergence of all states was higher than 0.05 significance level). Since the development of a biological system such as melanoma is a continuous spectrum, physical states are lumped to collective state vectors, thus enabling a succinct analysis of the biology. This same technique can be expanded to any number of other biological systems at varying levels of detail. A medical researcher in the field may be interested in the physiological changes of a system at the molecular level, thus encouraging the development of a Markov model to emulate these processes in the context of a larger biological system. Similarly, a practicing clinician may be more interested in overall patient response to a particular assay, thus encouraging the development of a Markov model to predict system response at a higher level of abstraction.

In each case, such a technique is extremely valuable to a system designer attempting to develop biomedical devices for these varied applications. Established techniques are generally available to provide biological system data in all but the most complex instances. Systems biologists and medical researchers can utilize this data to formulate simplified models of the highly complex biological system that, in turn, become a valuable asset to the design engineer. The intuitive nature of Markov models enables the engineer to not only design and simulate a system with stochastic biological components, but also to bridge the knowledge gap between complex biology and the engineering of complex biomedical devices (Tomlin 2007). In doing so, the validation, verification, and potential redesign of a physical system for experimental biomedical applications can be optimized to a point that is not currently possible using established system modeling techniques. 


\section{Implementation of Platforms for Experimental Biomedical Systems}

Utilizing the mechanisms available for the modeling of physical engineering systems and the techniques presented for the representation of biological systems using Markov models, a full platform for the engineering of experimental biomedical systems is achieved. Borrowing from the high-level system architecture model in figures 5 and 6, this framework platform creates a union of the two system domains that enables the simulation of a full biomedical system. Figure 8 showcases how such a union is achieved, where the biological element is modeled as a component in the system architecture.

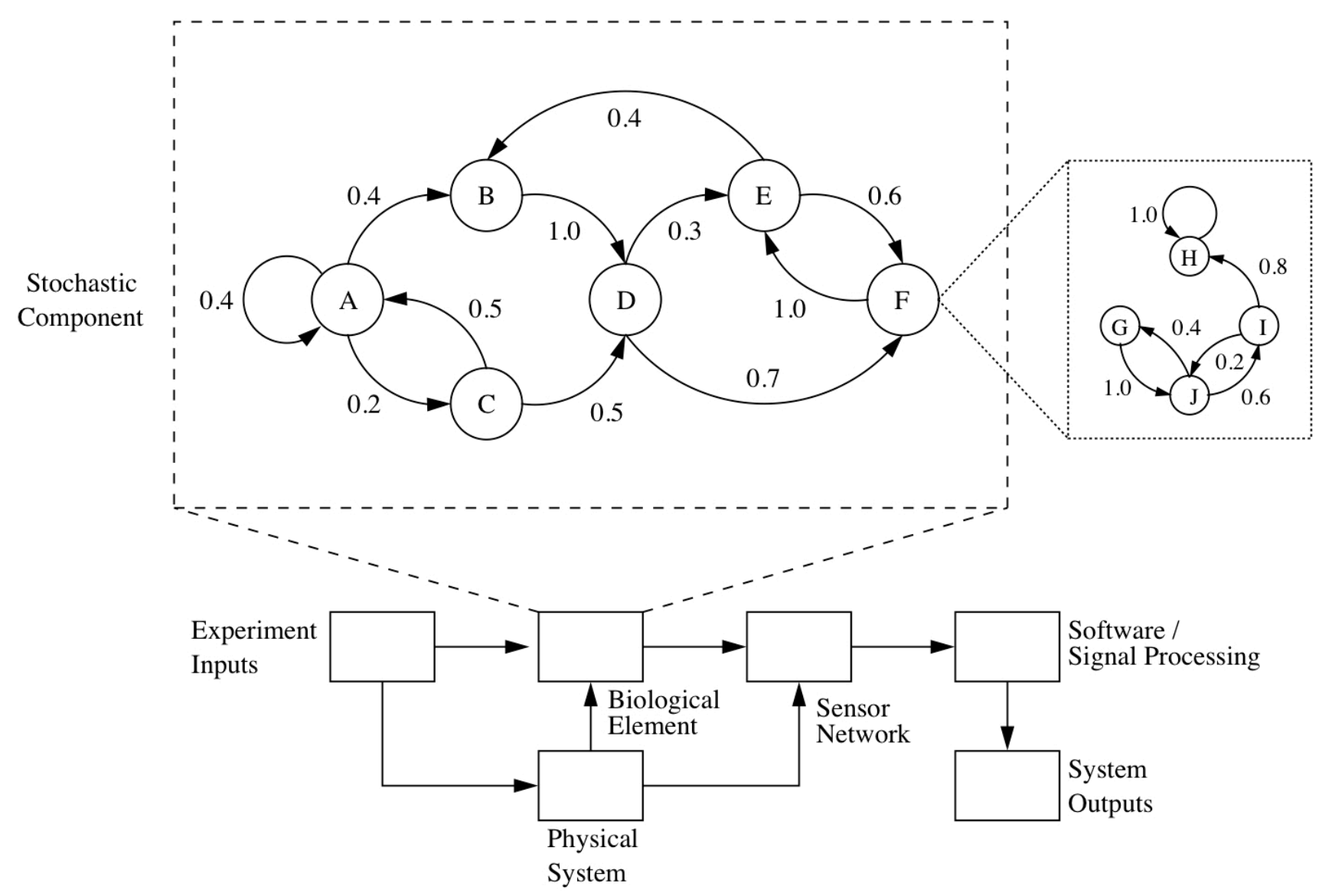

Figure 8. Implementation of the platform for engineering of experimental biomedical device systems

Modeling of the full system architecture is achievable using established systems modeling platforms, such as UML or SysML, since many of these have reached a level of maturity to support extensions to other languages and tools. In order to utilize the platform for overall engineering of the biomedical system, the implementation process follows a straightforward path as follows:

1. Gather relevant data of the biological system at the level of abstraction necessary for the application. This data will be used to formulate a Markov model of the biological system component. 
2. Formulate a Markov model of the biological component. An iterative process is often used to achieve convergence of such a model, as well as to define the appropriate segmentation of finite states for continuous systems (Kim 2002, Rabiner 1986).

3. Represent the validated Markov model using a tool that can integrate with the modeling platform used for the representation of the physical device system.

4. Design the proposed physical device components and how these components relate using the modeling platform named in (3). An additional component should also be represented in the system model that will extend to the biological component Markov model.

5. Perform simulation, validation, and verification of the complete system model. The results of these analyses will provide a means of redesign and device optimization for the particular experimental application.

The outputs generated from this system analysis will provide a range of potential experiment outputs based on the operation of the physical system and the stochastic biological system. The value of obtaining such a resultant set is paramount to the design engineer, as it allows them to directly address real-world concerns that would not otherwise be possible in the design phase. In the prototyping phase of device development and beyond, this same analysis can be used to verify proper device operation, to confirm the results of experiments, and to detect and avoid undesirable system performance. Such analyses are currently difficult and exceedingly timeconsuming using established methods, giving a platform for experimental biomedical device development considerable value to the entire medical field.

\section{Medical Drug Screening for Antibiotic Development}

This section presents a prototype application of the presented platform for engineering experimental biomedical systems. Medical drug screening for the development of new pharmaceutical drugs is a major area of concentration in the biomedical field. In order to develop methods of high-throughput screening for prospective antibiotics for treatment of bacterial infections, a microsystem was developed that is capable of arraying of experiments and non-invasive sensing.

The developed system contains all of the architectural components mentioned previously in this paper for experimental biomedical devices. A microfluidic platform provides a physical system module capable of containing the biological system. Additionally, a sensor network external to this microfluidic device enables continuous monitoring of bacterial development, where the cumulative outputs of the system can have a range of possibilities depending upon the stochastic biological system that the device is intended to analyze. Such architecture makes this application an ideal candidate for the use of the proposed platform, since reliance on the biological component makes system performance difficult to predict. 
The device itself is comprised of a microfluidic channel that is used to grow bacterial biofilms under controlled conditions. The biofilms are then treated with the candidate drugs in order to determine their levels of efficacy in depleting the bacterial films. Bacterial cultures, growth media, and the candidate antibiotics are supplied to the device via interface tubing, which allows for controlled system flow rates via an external syringe pump. Sensing of bacterial growth is achieved through optical density detection. As biofilm grows, it becomes increasingly absorbent to incident light (optically dense). Thus, biofilm growth can be monitored by tracking the amount of light transmitted through a biofilm sample. Sensing of this transmission is achieved via an array of photopixels placed underneath of the microfluidic growth chamber, where the analog voltage outputs of the pixels are inversely proportional to the biofilm optical density at that point. The advantage of this sensing mechanism is that it provides a means of non-invasive and continuous detection of biofilm growth that is otherwise difficult to obtain (Meyer 2011). Additional study of the biofilm is achievable through end-point measurements of density and morphology through the use of confocal microscopy. Figure 9 provides an overview of this architecture with the components of the system highlighted through images of the prototyped devices.

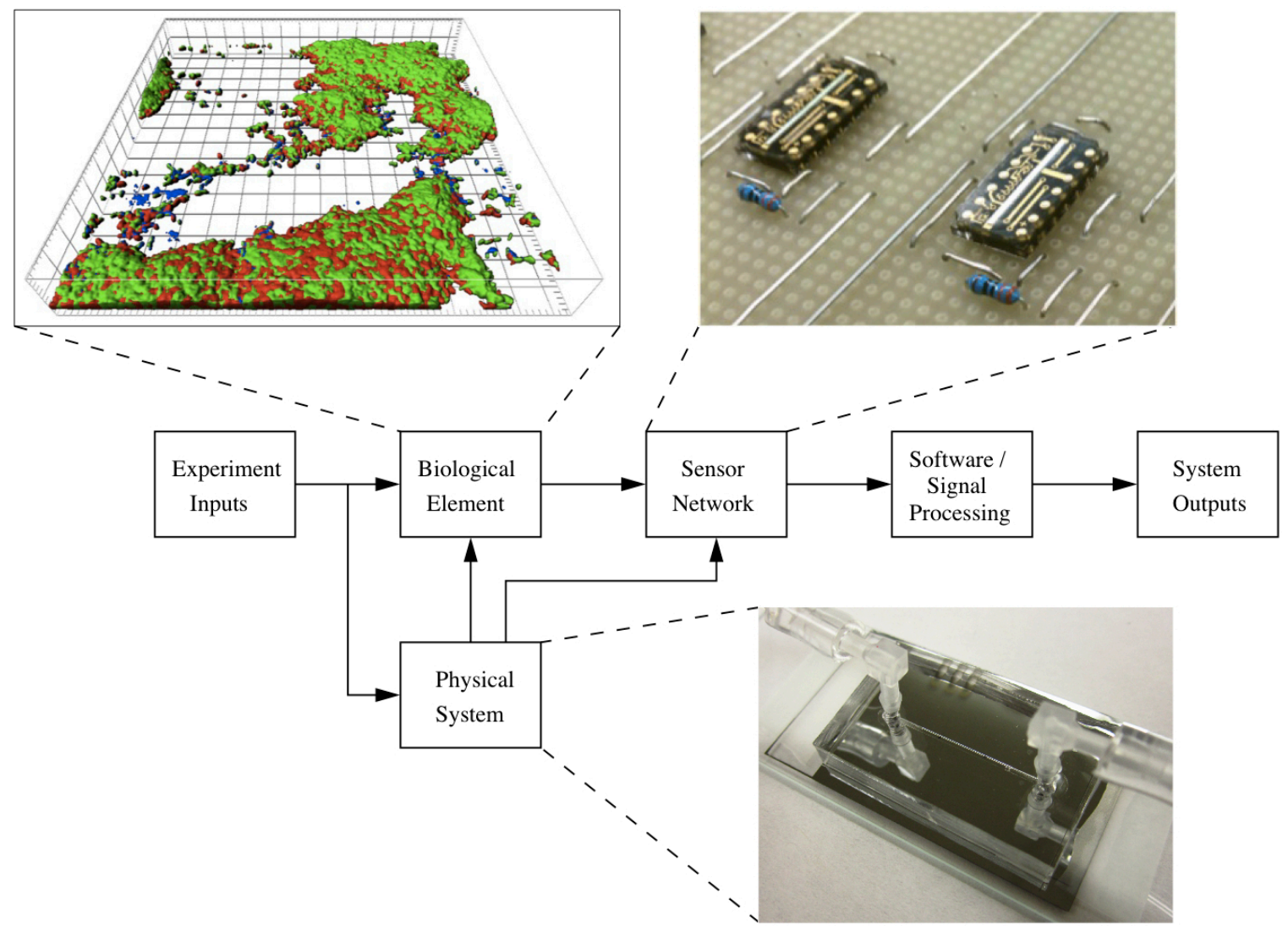

Figure 9. Bacterial imaging is performed using confocal microscopy and the microfluidic device is fabricated using soft lithography in polydimethylsiloxane (PDMS). The sensors are charge-coupled devices with 128x1 pixel arrays

To enable the formal validation and verification of this biomedical system, a Markov model of the bacterial biofilm component is developed. The current high-level model of the biofilm 
development process is implemented through the use of the tool presented by Yang. The software package enables the specification of a network of interacting Markov Chains, referred to as Markov Chain Cells in this paper, and of simulating the interactions of these Cells in order to investigate target characteristics of the network. Through reduction techniques utilizing symmetry in the Markov Chain network, highly complex models can be analyzed that would otherwise go beyond the computational capacity of most systems (Yang 2011).

The Markov Chain network used to describe this system is comprised of two distinct domains: the physical conditions of the experiment that affect the bacterial biofilm, and Markov Cells to describe the biofilm structure. Biofilm Markov Cells represent discrete sections of the film within the microfluidic chamber, where the state of each Cell is dependent upon the states of adjacent Cells as well as the states of the experimental conditions. Figure 10 shows the abstraction of the bacterial biofilm system as it is currently implemented, and follows directly from the architecture presented in figure 3. As this Markov model continues to mature, a clear path is to expand the model to a generalized two-dimensional biofilm with a suite of influencing experimental factors. Such advancements will permit the model's use in any number of design processes for biomedical devices intended for biofilm applications.

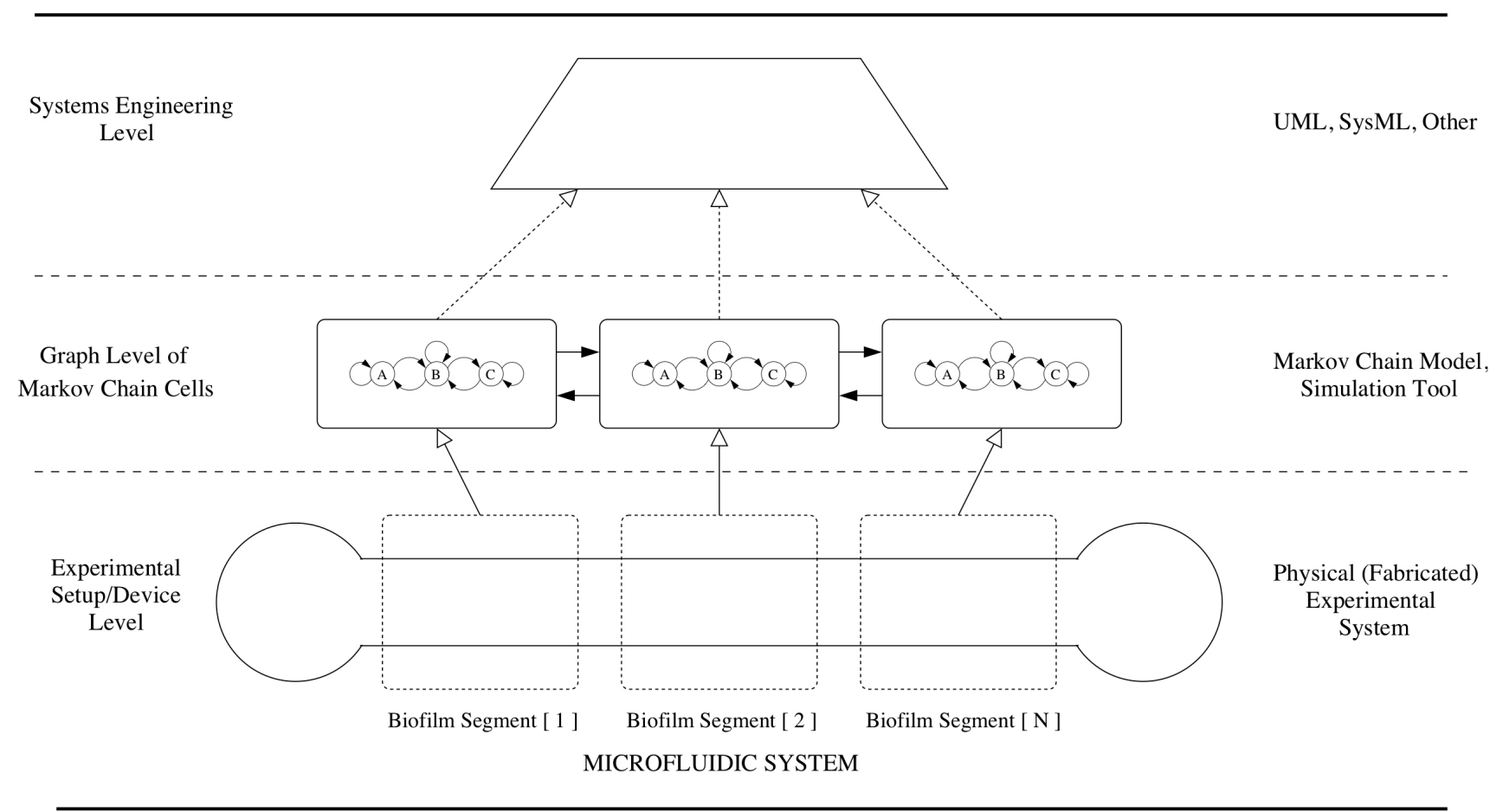

Figure 10. Visual representation of the biofilm Markov model implementation

An N-Cell biofilm was modeled with two key experimental condition variables: nutrient concentration in the system growth media and damaging shear stress due to fluid flow around the film. Each of these condition variables was provided binary values, and the biofilm elements were simplified to a system of three distinct states. The next-iteration state of each biofilm element is dependent upon its own Cell's current state, the current states of its adjacent Cells, and the current states of the experimental conditions. Overall, the number of possible states for 
the biofilm model was found to be $\mathrm{X}=2 * 2 * 3^{\mathrm{N}}$ according to Bayesian statistics (Yang 2011). Through the symmetry reduction methods, the system simulation was condensed from this set of possible states to a model with $0.75^{*} \mathrm{X}$ number of states, an overall $25 \%$ decrease in system complexity. By establishing an observer in the tool to track the number of biofilm Cells in various developmental stages, a full spectrum of theoretical biofilm growth characteristics is obtained that agrees with intuitive expectations (i.e. a near Gaussian profile). Future improvements to this model and its implementation in the simulation tool are expected to reduce this model even further, as previous implementations of its symmetric reduction principles have achieved orders-of-magnitude reduction in system size (Yang 2011).

\section{Conclusions and Future Work}

Conclusions. A platform for the design, validation, and verification of biomedical devices for experimental applications has been presented in this paper. The importance of this work stems from the current limitations of system modeling and analysis methods to consider systems with highly stochastic biological components. As a result, design engineers are incapable of developing ideal systems that properly address the device requirements of medical researchers and clinicians in the field. The effects of current limitations are further compounded by the knowledge disconnect that exists between biologists and engineers as a result of these shortcomings, since a lack of intuitive understanding of the biological component on the part of the engineer hinders proper system development. Here we have shown that the implementation of a platform for engineering experimental biomedical systems can bridge this gap and ensure more successful system design. By utilizing Markov Chain models to represent biological systems and extending these models to those of device components in established languages such as UML or SysML, an overall biomedical system model is achievable. A key benefit of this method is the enablement of formal system-level validation and verification of biomedical systems for experimental applications.

Future Work. In this paper, we define a revolutionary framework for the design and validation/verification of biomedical systems for highly stochastic experimental applications. To bring the benefits of such a platform to fruition, further work must explore methods to integrate tools for modeling biological systems with well-established modeling languages. Moving forward, such an architecture lays the foundation for a collaborative effort between biologists, clinicians, and systems engineers. Libraries of biological and device-oriented components achieved through this collaborative effort can be used in a broad number of application areas to develop new experimental systems for these disciplines.

\section{References}

Baldi, P., Chauvin, Y., Hunkapiller, T., McClure, M. A. "Hidden Markov Models of Biological Primary Sequence Information." PNAS 91 (3): 1059-1063.

Beck, J. R. 1983. "The Markov Process in Medical Prognosis." Medical Decision Making 4 (3): $419-458$.

Csete, M. E., and Doyle, J. C. 2002. "Reverse Engineering of Biological Complexity." Science 295: 1664-1669. 
Durbin, R., Eddy, S. R., Krogh, A., and Mitchison, G. 2002. Biological Sequence Analysis: Probabilistic Models of Proteins and Nucleic Acids, 46 - 159. Cambridge (UK): Cambridge.

Endy, D. 2005. "Foundations for Engineering Biology.” Nature 438 (24): 449-453.

Janakiraman, V., Englert, D., Jayaraman, A., and Baskaran, H. 2009. "Modeling Growth and Quorum Sensing in Biofilms Grown in Microfluidic Chambers." Annals of Biomedical Engineering 37 (6): 1206-1216.

Kim, S., Li, H., Dougherty, E. R., Cao, N., Chen, Y., Bittner, M., Suh, E. B. 2002. "Can Markov Chain Models Mimic Biological Regulation?” Journal of Biological Systems 10 (4): 337357.

Meyer, M. T., Roy, V., Bentley, W. E., and Ghodssi, R. 2011. "Development and Validation of a Microfluidic Reactor for Biofilm Monitoring Via Optical Methods." Journal of Micromechanics and Microengineering 10: 1-10.

Oltvai, Z. N., and Barabasi, A. L. 2002. "Systems Biology. Life's Complexity Pyramid." Science 298: 763-764.

Potera, C. 1999. "Microbiology - Forging a Link Between Biofims and Disease." Science 283: 1837-1839.

Rabiner, L. R., Juang, B. H. 1986. "An Introduction to Hidden Markov Models." IEEE Acoustics, Speech, and Signal Processing 3 (1): 4-16.

Richards, J. J., and Melander, C. 2009. "Controlling Bacterial Biofilms." Chemistry and Biochemistry 10: 2287-2294.

Tomlin, C. J., and Axelrod, J. D. 2005. "Understanding Biology by Reverse Engineering the Control." PNAS 102 (12): 4219-4220.

Tomlin, C. J., and Axelrod, J. D. 2007. "Biology by Numbers: Mathematical Modelling in Developmental Biology." Nature Reviews; Genetics 8: 331-340.

Van Hulst, R. 1978. "On the Dynamics of Vegetation: Markov Chains as Models of Succession." Theory and Models in Vegetation Science 40 (1): 3-14.

Yang, S., Zhou, Y., Baras, J. 2011. "Compositional Analysis of Dynamic Bayesian Networks and Applications to CPS." Paper submitted to the Third International Conference on Cyber-Physical Systems, Beijing, China, 17-19 August 2012. [Submitted]

\section{Biography}

Matthew Mosteller is a graduate student in Systems Engineering at the Institute for Systems Research at the University of Maryland, College Park. He has a Bachelors degree in Electrical Engineering from the University of Maryland, College Park.

Mark Austin is an Associate Professor in the Department of Civil and Environmental Engineering, University of Maryland, College Park. He currently holds an affiliate appointment with the Institute for Systems Research. During the past ten years, Mark has taught extensively in the Master of Science in Systems Engineering (MSSE) program, and conducted short courses in Systems Engineering at US companies, and in Europe and South America. $\mathrm{He}$ has a Bachelors degree in Civil Engineering from the University of Canterbury, New Zealand, and Masters and Ph.D. degrees in Civil Engineering from UC Berkeley. 
Reza Ghodssi is the Herbert Rabin Distinguished Professor, Director of the Institute for Systems Research (ISR), and Director of the MEMS Sensors and Actuators Lab (MSAL) in the Department of Electrical and Computer Engineering (ECE) at the University of Maryland. His research interests are in the design and development of microfabrication technologies and their applications to micro/nano devices and systems for chemical and biological sensing, small-scale energy conversion and harvesting. He has a Bachelors, Masters, and Ph.D. in Electrical Engineering from the University of Wiscosin - Madison.

Shah-An Yang is a post-doctoral associate in Systems Engineering at the Institute for Systems Research at the University of Maryland, College Park. He has a Bachelors degree in Electrical Engineering and a Ph.D. in Electrical Engineering from the University of Maryland, College Park. 